\title{
DOUBLE STAR OBSERVATIONS WITH HIPPARCOS: reduction methods and early results by NDAC
}

\author{
S. Söderhjelm ${ }^{1}$, D.W. Evans ${ }^{2}$, F. van Leeuwen ${ }^{2}$, L. Lindegren ${ }^{1}$
}

${ }^{1}$ Lund Observatory, Box 43, S-221 00 Lund, Sweden

${ }^{2}$ Royal Greenwich Observatory, Madingley Road, Cambridge CB3 0EZ, UK

\section{ABSTRACT:}

The Hipparcos satellite has a predefined observing program given by an Input Catalogue with 118000 entries. This includes some 10000 members of known double or multiple systems (with separations above 0.1 arcsec and magnitude-differences below 4-5), which are observably non-single and thus need a more complex reduction procedure. Also, a similar number of hitherto unknown doubles showing deviations from a single-star observation model will be included in the double-star reductions. A key feature in the NDAC reductions is the use of the main reduction results to calibrate and collect the data for individual doubles in 'Case History Files'. This enables a global solution of both absolute and relative parameters for a double to be carried out later in a single solution. Using provisional data tapes covering small parts of a 14-month interval, tests have been made of major parts of the double star reductions. Comparisons with the main reduction results for single stars indicate that remaining calibration errors are below the 10 milliarcsec level. For known doubles, the solutions are good relative to the Input Catalogue at the expected 0.3 arcsec level, and for a few tens of systems observed with the starmapper, the agreement is at the 0.05 arcsec level. A few hundred doubles not flagged in the Input Catalogue have also been detected and had their parameters provisionally determined. A detailed paper describing these methods and results has been accepted by Astronomy \& Astrophysics. 